\title{
A Context-Aware Intelligent System to Introduce Mobile-Cloud Services for Diabetes Management
}

\author{
Asmae Benali ${ }^{1}$, Bouchra El Asri ${ }^{2}$ \\ I(IMS Team SIME Laboratory ENSIAS, Mohamed V University, Morocco) \\ ${ }^{2}$ (IMS Team SIME Laboratory ENSIAS, Mohamed V University, Morocco)
}

\begin{abstract}
The healthcare sector is one of the most important sectors in all countries where it spends an average of about $9.5 \%$ of its domestic product. Diabetes is a chronic disease that demands a long term healthcare; it consumes medical resources, requires regular set of checkups and continual requirements for physicians and medical equipment. In addition, the patient should control blood-glucose levels with a normal range through medication, diet and exercise. In consequence, the majority of patients suffer from their incapability to manage their treatments without difficulty and the boring follow-up process. In this paper, we propose a novel framework that combines mobile and cloud computing with software product line techniques in order to offer real-time and context-aware services that take into consideration the variability of both the context of the patient to get a fresh data and the cloud environment to provide a service that satisfies the user requirements.
\end{abstract}

Keywords - Context-aware System, E-health, Diabetes, Diabetes Management System, Mobile Cloud Computing, Software Product Lines

\section{Introduction}

Diabetes is considered as an incurable disease that affects around 194 million people worldwide according to the World Health Organization and it foresee the increment of that number by 2015 to at least 300 million [1]. In the developed countries, diabetes has evolved into the fourth main cause of death. In fact, according to the New York Time's magazine, developing countries suffer from a huge percentage of Diabetes patients that arrives at almost $42 \%$ of the population. Many of these patients face early-stage eye disease, whereas about $5 \%$ are completely blind [2].

Medical statistic depicted that the rate of diabetes in Africa was equal to or inferior to $1.4 \%$ from 1959 to the mid-1980s , except South Africa, where the rate was estimated to be as high as 3.6\% in 2001[3][4]. However, the continent-wise prevalence of diabetes mellitus, by 1994, expected to 3 million and was, by the year 2010, predicted to double or triple [5][6]. By 2030, this number was expected to increase to 18,6 million[7]. No supervision of the diseases not only ensues long term complications, as blindness and stroke, but can more than that make the diabetic at immediate danger.

In several developing and newly industrialized nations, diabetes is attaining epidemic proportions [8]. These high numbers are principally owing to the bad and wrong management of the diseases. In order to prevent the impacts and the complications of diabetes, it must hold it continually under regular control.

The majority of diabetic patients find the treatment processes boring, as the patient should measure daily the blood-glucose and blood- pressure levels, make regular check-ups, take medications, follow-up diet and exercise regularly, keep their record and visit their physician. Consequently, patient by time can neglect his daily treatment.

These days, mobile devices as mobile phones are passing into necessary [9], where they become the main sources of information for users, as they can be used easily, adopt recent information technologies with modern communication technologies, offer a lot of valuable utilities. Having a mobile application that maintains and manages diabetes treatment can provide an efficient healthcare service quality. Especially, when it takes into consideration the encouragement and the participation of the both the family and the healthcare professionals to ameliorate patient self-care. In fact, an interactive system for patients and caregivers is a useful way to protect the life of the patient by encouraging him to be independent and punctual.

Here, we propose a context-aware and intelligent mobile and cloud based system for diabetes efficient management. The proposed system helps the patient to acquire his diabetes measurement, save his records, reminds him about check-ups date and medications times. In addition, it gives the patient the guide in case of increase or decrease of blood glucose level, so well as saving his life in emergency state.

The rest of this paper is organized as follows. In section 2, we present the background on the diabetes disease and some used technologies. Section 3 compares and positions our solution with other proposals found in the literature. Section 4 illustrates our proposed solution. Finally, we conclude and present our future work in Section 5. 


\section{Background}

In this section, we define some of the concepts related to the diabetes, its data management systems and software product lines engineering.

\subsection{Diabetes Mellitus}

Diabetes is the result of the incorrect use of the glucose either the insufficient production of insulin by the pancreas or the wrong reaction of the cells to the insulin produced. Diabetes is divided into three main types; type I, type II and Gestational diabetes. Many patients can suffer from the short and long term complications of this disease, which are the result of the bad management of diabetes and other associated health problems. The maintenance of a healthy lifestyle, supervision of blood glucose and pressure, regular check-ups can protect the patients of these problems [10]. As reported by the International Diabetes Federation [11], in the world, at least 285 million people are affected by diabetes and that number is estimated, by the year 2030, to arrive 438 million, with two-thirds of all diabetes cases taking place in low-to middle-income countries. In fact, high percentage of diabetic patients is founded in the countries witch miss to the needed infrastructures for diabetes care because of the bad management of this disease.

\subsection{Diabetes Mobile-based Management Systems}

Management of diabetes has become easy due to the divers developed technologies. Many mobile phones incorporate other features like high quality cameras, recording devices and personal management tools. The use of connectivity in mobile phones; combining messaging, emailing and GPS locating, permits to create advanced utilities to rescue patient life by holding him connected with his physician and caregivers. Testing blood glucose at home was a major jump for diabetics. When the patient finish from this test, he manage system records and stores the test result as well as the other related and important data such as; the type and dosage of insulin date, time of tests, etc.

The obtained data can also be modeled as a chart so as to give the patient the possibility to apparently pursue the progress of his blood glucose.

\subsection{Cloud computing and Diabetes Management}

The NIST (National Institute of Standards and Technology) has defined cloud computing as a model that enable convenient, on-demand access to a shared pool of configurable computing resources (e.g., networks, servers, storage, applications, and services) which can be quickly provided with minimal management effort and interaction [12]. Cloud computing gives its clients the possibility to store and process their data in data centers with diverse capabilities.

Mobile device is still a constrained resource (resource poverty, battery constraint), in particular healthcare applications that requires more resources than mobile device can offer. Hence, the necessity of an external source as cloud computing platforms. Healthcare providers are looking at automated processes with reduced cost and high gains, cloud computing can present an ideal solution for the healthcare IT space. Hospitals can use infrastructure in common with large number of systems connected together. Consequently, the hospitals reduce automatically the cost and step up the use. In other side, the resources are transferred only under demand, which means real-time availability of patient information for medical care institutions and other personnel support utilities from any device that enables internet.

The main cause of the bad management of diabetes is the decentralized data that are saved in different places. Hosting all important information of diabetic person in one location and make it accessible anywhere, the patient, the doctors and the patient family can have an integral view of everything that influences the blood glucose levels and can then participate in a considerable enhancement in blood glucose control. The access and the management of diabetes over internet can certainly improve the patient engagement and aid him to stay more linked to his self-care; in particular the patients who live in remote areas which suffer from no availability of healthcare assistance.

\subsection{Software Product Lines}

In recent years, software product lines (SPL) approach has been used in various research areas due to its reusability aspect that allows optimizing the product development. In fact, this approach relies on the reuse of common elements that are similar in many products through the software development process. Consequently, it provides a major gain in resources, time and quality [13]. Software Product Line Engineering (SPLE) is composed of two main processes; domain engineering and application engineering. The first process concerns the creation of assets which can be a model, document, software component or any other unit that participates in the development of system. According to the second process has as mission the construction of the final products taking into account the particular exigencies.

Variability in software product lines can be an essential and technical variability [14], internal and external variability [15], product line and software variability [16]. Variability modeling permits to identify and 
construct reusable assets which can be employed to build novel products minimizing the effort, time and cost spent in the building from scratch. To model the variability, feature diagrams notion was proposed by Kang et al. in 1990 [17]. Indeed, feature diagram permits the modulation of variability among a set of identical products; where the commonalities and variabilities among products in the same domain are illustrated as features. A feature is a prominent or distinctive aspect, quality, or characteristic of a software system [18]. A feature can be either [19] i) Mandatory, it is presented in all products if the feature parent is selected; ii) Optional, it is not identified in all products; iii) Alternative, it specifies more general feature; only one option can be picked from several features iv) Or: One or more features can be identified in the product.

\section{Related Work}

Mobile phone has become currently the main sources of information and many applications have been developed in different mobile operation systems to satisfy the user requirements. Many applications have been created to handle and manage diabetes such as Glucose Buddy, BGluMon, WaveSense and Vree.

BGluMon (Blood Glucose Monitor) is a mobile application that gives the patient the possibility to see plainly and daily his blood glucose concentration by including recording and exporting data functions; it is downloadable on Apple Store [20].

Glucose Buddy is an application found in Apple Store that permits the management of patient's logs like, blood glucose level, weight, medication, exercise and food. The results are presented in the form of grid table which lacks for analysis [21].

For measuring blood glucose level, WaveSense application includes a meter that can be linked to iphone or any other Apple device. To determine the blood glucose level, WaveSense adopt strips specific for this meter and after the measurement of blood glucose level, data is automatically stored in the WaveSense application [22]. This meter and its strips are not available in all countries, whence the utilization of this application is limited.

Vree is a mobile application for Apple iOS that is done for type II diabetes. This application contains several functions and calculations, however, they cannot be exploited for the other types of diabetes [23].

A suggested telemonitoring system for patients to help them to send blood glucose and blood pressure data to the system database. Subsequently, the physician could access to the database to examine patient data as well as generate the patient report [24]. But, this system aims attention at the transmission of multimedia data from the hospital's database, and it does not permit direct communication among patients, medical care institutions and caregivers.

On Track is a mobile application found in Android Marker that is developed in order to treat and manage diabetes through tracking the level of glucose in the blood, medication, food, exercise, weight. The application due to these tracked values can give test and medication values in form of table as well as a resume about the patient progress and his history [25]. However, On Track application misses some utilities; such as connectivity.

At present, the available mobile applications took into account some functionality; however, they are limited in many others which are important to increase the patient improvement level.

\section{Proposed Solution}

In this section, we introduce a context-aware system that combines mobile computing and cloud computing basing on data mining and software product lines techniques so as to establish a smart and interactive system for diabetes management.

\section{Beneficiaries}

We identify in this section the beneficiaries and contributors in the proposed system. We decline afterward their different behaviors and interactions with the system.

- Diabetic Patient: patient gives and stores using the mobile application their basic information about its profile; such as the name, date of birth, address, gender, personal medical record, and other related genetic records. In addition, the patient can provide all information that relate to the disease as blood glucose and pressure values, measurement scenario, check-ups, medications, caregivers contact. In other side, the patient can follow a diet by giving the daily information that concerns food and exercise.

- Health professionals: represent doctors, nurses, radiology centers, workers at laboratory that participate in the medical record management fulfilled by the patient. This unity modify the record by including medical information and store it afterwards as an electronic health record in the cloud and make it accessible to other health professionals in diabetes.

- Caregivers: is the patient family member or other person that takes care of the patient. In Emergency case, the system notify the caregivers about the patient measurement status and in critical situation, it send them a signal that give the location of the patient. 
- Government: When the data is analyzed with elevated refresh rates collected from the country population, the obtained reports about diabetes distribution aid at efficient resources reallocation.

\section{System Architecture}

Fig. 1 shows the overall presentation of the system architecture of our proposed mobile healthcare system for diabetic.

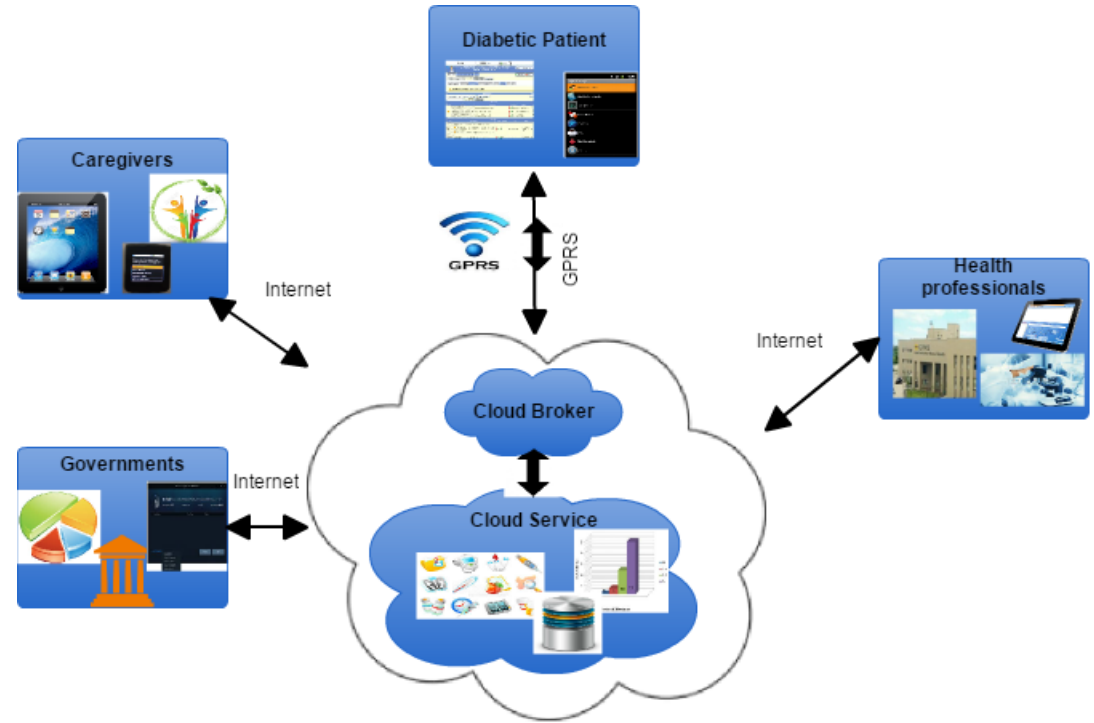

Fig. 1. System architecture.

Our system is based on two techniques; data mining and software product lines engineering. In fact, data mining techniques and discovery algorithms are an efficient method to process patient health record and feedbacks in order to provide a fresh and smart data. While, software product lines approach is used to cope with the variability of the patient context; such as location, health status as well as the variation of cloud service environment, like allocation resources, quality of service, etc..

In this paper, we suggest a context-aware and interactive system that incorporates between tow wide and main technologies; mobile phones and cloud computing, basing on data mining techniques and on largely software product lines engineering. In other side, our system repose on General Packet Radio Service (GPRS) blood-glucose monitor (BGM) that is an Android device permitting two way communication. GPRS BGM is available in the market and conforms to Health Insurance Portability and Accountability [26] and ISO 15197 [27]. Hence we have exploited this device to set up communication between diabetic patients and his family or caregivers. The system gathers patient information by means of the GPRS BGM; as blood glucose values, blood pressure, measurement scenarios, location and times (before/after exercise, before/after meals, at day/night...).

Due to data mining techniques and knowledge discovery algorithms, the acquired data are processed and treated in order to obtain a smart and fresh data. Therefore, the system uploads the data to cloud broker via the GPRS protocol and XML format. Accordingly, the system illustrates the obtained patient information as a feature model and generates the XML-format that corresponds to this representation (see Fig. 2).

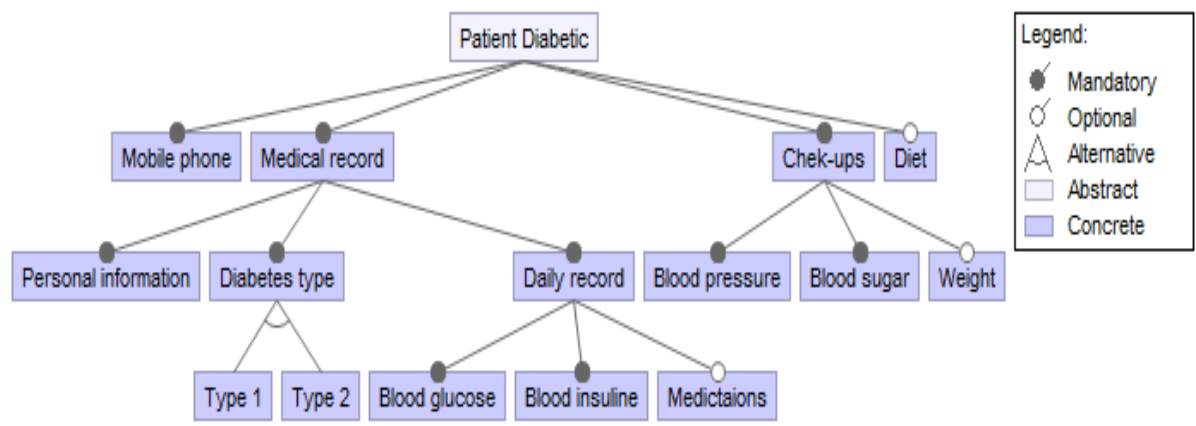

Fig. 2. Diabetic patient - feature model.

After the patient information analyzing, the system detects the health status of the patient and other required services that concern the location of the patient, the security type to be applied and other aspects that allow to efficiently reallocating medical resources. In other hand, in accordance with this information and 
features selected, the service provided will be presented as a feature model and select afterwards the features that take part of the service provisioned by the concerned cloud provider (see Fig. 3).

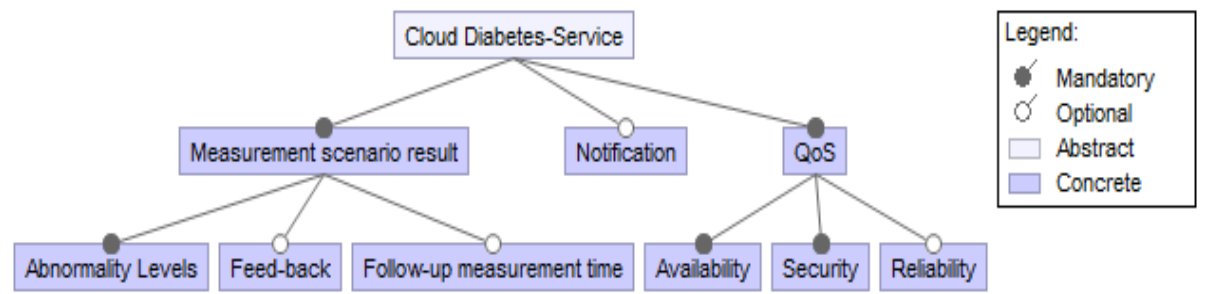

Fig. 3. Cloud service- feature model.

Moreover, the system determines the blood level as a feed-back or abnormality level according to the important related patient information and measurement scenario as showed in Fig 4. In health status in which the diabetic patient is critical, the system will send automatically a notification to patient caregivers via the Message Queue Telemetry Transport (MQTT) protocol. Indeed, the MQTT can send a real-time alarm to a caregiver about the measurement values and in case of emergency it transmits the diabetic location signal in order to save his life.

ID $\longleftarrow$ Patient identifier in the system

$\mathrm{BCF} \longleftarrow$ Blood glucose feature

$\mathrm{DTF} \longleftarrow$ Diabetes type feature

$\mathrm{ALF} \longleftarrow$ Abnormality level feature

ALC $\longleftarrow$ Current blood glucose abnormal level

ALL־Abnormal level of last measurement

$\mathrm{MsC} \longleftarrow$ current message of measurement scenario

$\mathrm{MsL} \longleftarrow$ Last message of measurement scenario

$\mathrm{Ntf} \longleftarrow$ Notification feature

$B G M_{I D}$ the GPRS BGM number of diabetic ID

M $\longleftarrow$ Telecare message of current measurement

$\mathrm{R} \longleftarrow$ Reminder

Fct $\longleftarrow$ Measurement scenario result feature

Function AB_level_detect(int ID, string DTF, int BCF)

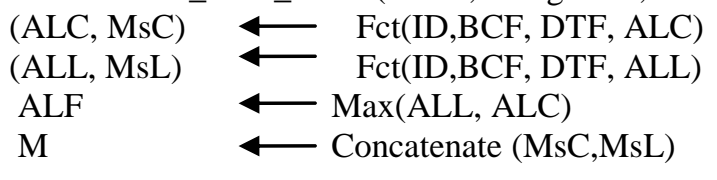

if $(\mathrm{ALF}==0)$

push(Ntf, $\left.B G M_{I D}\right)$

else if $(\mathrm{ALF}==1)$

$\operatorname{push}\left(\mathrm{Ntf}, B G M_{I D}\right)$

else

push(Ntf, $\left.B G M_{I D}\right)$

$\operatorname{push}(\mathrm{Ntf}, \mathrm{R})$

end if

MQTT_Message(ALF,M, Ntf)

End Function

Fig. 4. The algorithm for blood glucose level detection based on feature model. 


\section{Conclusion}

Applying cloud computing and mobile device in diabetes management, it is possible to change patient life-style to better. The elasticity, scalability, virtualization, and distributed characteristics of cloud computing could aid at the coordination and exchange information more efficiently. In other hand, mobile phones make the management of this disease more easily due to the developed technologies that they include.

We proposed in this paper a detailed healthcare framework for diabetes management which associates between smart phones and cloud computing technologies. This framework is based on software product line so as to manage the variability of patient context; like measurements scenario, location, and daily record. In addition, it takes into consideration environment changing of the services engendered by the provider; such as the blood level detection which relies on the patient's information received, availability of the resources, security, etc...

Further, we plan to develop interactive mobile applications that cover other endemic diseases by exploiting our proposed framework.

\section{References}

[1] Diagnosis and Classification of Diabetes Mellitus, Diabetes Care, vol. 34, no. 1, 2011, pp. S62-S69; doi:10.2337/dc11-S062.

[2] Hamdan, S.: Rapid Increase of Diabetes Strains Middle East's Health Agencies. New York Times, The International Herald Tribune Section, 2011.

[3] Motala, AA.: Diabetes trends in Africa. Diabetes Metab Res Rev. 2002;18:S14-20

[4] Rheeder, P. : Type 2 diabetes: The emerging epidemic. South Afr Fam Pract. 2006;48:20.

[5] Sobngwi, E. , Mauvais-Jarvis, F., Vexiau, P., Mbanya, JC., Gautier, JF. : Diabetes in Africans: Part 1: Epidemiology and clinical specificities. Diabetes Metab. 2001;27:628-34.

[6] International Diabetes Federation. Diabetes Atlas. 2nd ed. Belgium: IDF, Brussels; 2003.

[7] Wild, S., Roglic, G., Green, A., Sicree, R., King, H.: Global prevalence of diabetes: Estimates for the year 2000 and projections for 2030. Diabetes Care. 2004;27:1047-53.

[8] Gan, D.: editor. Diabetes atlas, 2nd ed. Brussels: International Diabetes Federation; 2003. http://www.eatlas.idf.org/webdata/docs/Atlas\%202003-Summary.pdf (accessed June 19, 2007).

[9] http://en.wikipedia.org/wiki/Mobile_Phones. Wikipedia, "Mobile phone - Wikipedia, the free encyclopedia," Accessed March 2010.

[10] Diabetes: Available from https://www.diabetes.org.uk/Guide-to diabetes/Complications/, Accessed 1 November 2016.

[11] International Diabetes Federation. IDF Diabetes Atlas. Epidemiology and Mobility: Available from http://www.idf.org/. Accessed on 5 November 2016.

[12] Mell, P., Grance, T.: The NIST definition of cloud computing (v15). National Institute of Standards and Technology, Tech. Rep., 2009.

[13] Clements, P., Northrop, L.: Software Product Lines: Practices and Patterns. Addison-Wesley Longman Publishing Co., Inc., 2001.

[14] Halmans, G., Pohl, K.: Communicating the variability of a software product family to consumers. Software and Systems Modeling, 2(1):15-36, 2003

[15] Pohl, K., Böckle, G., van der Linden, F. J. : Software Product Line Engineering: Foundations, Principles and Techniques. SpringerVerlag New York.

[16] Metzger, A. , Pohl, K., Heymans, P., Schobbens, P., Saval, G. : Disambiguating the Documentation of Variability in Software Product Lines: A Separation of Concerns, Formalization and Automated Analysis.

[17] Kyo, C. K., Sholom, G. C., James, A. H., William, E. N., Peterson, A.S.: Feature-oriented domain analysis (foda) feasibility study. Technical report, Carnegie-Mellon University Software Engineering Institute, November 1990.

[18] Kang, K.C., Cohen, S.G., Hess, J.A., Novak, W.E., Peterson, A.S.: Feature-oriented domain analysis (FODA) feasibility study. DTIC Document, 1990.

[19] Gurp, J.V., Bosch, J., Svahnberg, M.: On the notion of variability in software product lines. In Proceedings of the Working IEEE/IFIP Conference on Software Architecture, pp. 45, 2001.

[20] "bglumon,".Available from: http://itunes.apple.com/us/app/bglumonblood-glucosemonitor/id353118307?mt=8, Accessed 30 November 2016.

[21] Glucose buddy. Available: http://www.glucosebuddy.com/. Accessed 30 November 2016.

[22] IBG Star: Available from: http://www.ibgstar.us/default.aspx. Accessed 30 November 2016.

[23] "Vree," Apple Store: Available from http://itunes.apple.com/us/app/vree-fordiabetes/id355923059?mt=8. Accessed 30 November 2016.

[24] Wakefield, B.J., et al.,: Effect of Home Telemonitoring on Glycemic and Blood Pressure Control in Primary Care Clinic Patients with Diabetes. Telemedicine J. and E-Health, vol. 20, no. 3, 2014, pp. 199-205; doi:10.1089/tmj.2013.0151.

[25] "On Track," Android Market: Available from:https://play.google.com/store/apps/details?id=com.gexperts.ontrack\&hl=en .Accessed 30 November 2016.

[26] Dwyer III, S.J., et al.: Health Insurance Portability and Accountability Act. Security Issues in the Digital Medical Enterprise, vol. 72, no. 2, 2004, pp. 9-18.

[27] ISO 15197:2013-In Vitro Diagnostic Test Systems - Requirements for Blood-Glucose Monitoring Systems for Self-Testing in Managing Diabetes Mellitus, ISO standard, 2013. 